Statistics

\title{
Rates of convergence for nonparametric deconvolution
}

\author{
Claire LACOUR ${ }^{\mathrm{a}}$, \\ ${ }^{a}$ Laboratoire MAP 5, Université Paris 5, 45 rue des Saints-Pères, 75270 Paris Cedex 06
}

\begin{abstract}
This Note presents original rates of convergence for the deconvolution problem. We assume that both the estimated density and noise density are supersmooth and we compute the risk for two kinds of estimators.

\section{Résumé}

Vitesses de convergence en déconvolution nonparamétrique. Cette Note présente des vitesses de convergence originales pour le problème de déconvolution. On suppose que la densité estimée ainsi que la densité du bruit sont « supersmooth » et on calcule le risque pour deux types d'estimateurs.
\end{abstract}

Version française abrégée

On considère le problème de déconvolution suivant :

$$
Y_{i}=X_{i}+\varepsilon_{i} \quad i=1, \ldots, n
$$

où les $X_{i}$ sont des variables aléatoires indépendantes et identiquement distribuées de densité inconnue $g$ et les variables aléatoires $\varepsilon_{i}$ sont i.i.d de densité connue $f_{\varepsilon}$. Les suites $\left(X_{i}\right)$ et $\left(\varepsilon_{i}\right)$ sont de plus supposées indépendantes. L'objectif est d'estimer $g$ à partir des données $Y_{1}, \ldots, Y_{n}$.

Le cadre d'hypothèses est le suivant. Notons, pour toute fonction $u, u^{*}$ la transformée de Fourier de $u: u^{*}(x)=\int e^{i x t} u(t) d t$. On suppose que le bruit est tel que pour tout $x$ de $\mathbb{R}, f_{\varepsilon}^{*}(x) \neq 0$ et qu'il satisfait l'hypothèse suivante :

N1. Il existe $s \geq 0, b \geq 0, \gamma \in \mathbb{R}(\gamma>0$ si $s=0)$ et $k_{0}, k_{1}>0$ tels que

$$
k_{0}\left(x^{2}+1\right)^{-\gamma / 2} \exp \left(-b|x|^{s}\right) \leq\left|f_{\varepsilon}^{*}(x)\right| \leq k_{1}\left(x^{2}+1\right)^{-\gamma / 2} \exp \left(-b|x|^{s}\right)
$$

Email address: lacour@math-info.univ-paris5.fr (Claire LACOUR). 
On suppose de plus que $g$ appartient à l'espace

$$
\mathcal{A}_{\delta, r, a}(L)=\left\{g \text { densité sur } \mathbb{R} \text { et } \int\left|g^{*}(x)\right|^{2}\left(x^{2}+1\right)^{\delta} \exp \left(2 a|x|^{r}\right) \leq L\right\}
$$

avec $r \geq 0, a \geq 0, \delta \in \mathbb{R}(\delta>1 / 2$ si $r=0), L>0$. Lorsque $r$ est strictement positif, la fonction est dite superrégulière et ordinairement régulière sinon, la terminologie étant la même pour le bruit.

Ce problème a été largement étudié pour une fonction $g$ appartenant à un espace de Sobolev ou de Hölder (i.e. $r=0$ ) : on peut citer entre autres [3], [5], [6,7], [8], [10]. Les vitesse de convergences médiocres (en puissances de $\ln n$ ) obtenues lorsque le bruit est superrégulier (et en particulier pour les distributions gaussiennes) ont conduit à considérer des fonctions $g$ également superrégulières. En premier [9] et plus récemment [1], [2] et [4] ont étudié des estimateurs dans ce contexte.

Dans cette Note, nous fournissons des vitesses de convergence exactes et explicites, même dans le cas $r>0, s>0$ pour lequel jusqu'à maintenant les vitesses n'étaient données que de façon implicite, excepté dans des cas très particuliers. Ces vitesses sont calculées pour deux types d'estimateurs.

L'estimateur à noyau classique est le suivant :

$$
\hat{g}_{n}(x)=\frac{1}{n h} \sum_{i=1}^{n} K\left(\frac{x-Y_{i}}{h}\right)
$$

où $K$ est défini comme la transformée de Fourier inverse de $K^{*}(x)=\mathbb{1}_{\{|x| \leq 1\}} / f_{\varepsilon}^{*}(x / h)$. Le risque ponctuel (noté MSE) et le risque intégré (notée MISE) sont établis dans [2] (voir la proposition 2.1 ci-dessous). Cet estimateur a l'avantage d'être optimal au sens minimax asymptotique fin (voir [2]) mais ne fournit d'estimateur adaptatif que dans des cas particuliers. C'est pourquoi nous nous intéressons également à l'estimateur de projection introduit dans [4].

Soit $\varphi(x)=\sin (\pi x) /(\pi x)$ et $\varphi_{m, j}(x)=\sqrt{L_{m}} \varphi\left(L_{m} x-j\right)$. En posant $u_{t}(x)=(1 / 2 \pi)\left(t^{*}(-x) / f_{\varepsilon}^{*}(x)\right)$, l'estimateur de projection est défini par

$$
\hat{g}_{m}(x)=\sum_{|j| \leq K_{n}} \hat{a}_{m, j} \varphi_{m, j} \quad \text { où } \hat{a}_{m, j}=\frac{1}{n} \sum_{i=1}^{n} u_{\varphi_{m, j}}^{*}\left(Y_{i}\right)
$$

Pour cet estimateur, le calcul du rique intégré est présenté dans [4] (voir la proposition 2.2 ci-dessous). Un estimateur adaptatif peut ensuite être défini en utilisant une technique de sélection de modèles (voir [4] pour les détails).

On peut observer que les deux estimateurs ont les mêmes vitesses de convergence, qui s'obtiennent en minimisant l'ordre du risque. On est alors ramené à résoudre l'équation suivante

$$
\exp \left(\frac{2 b}{h^{s}}+\frac{2 a}{h^{r}}\right) h^{\alpha}=O(n)
$$

où $\alpha=r-2 \delta-2 \gamma-1$ lorsque l'on considère l'erreur intégrée et $\alpha=-2 \delta-2 \gamma+(s-1)_{+}$lorsque l'on considère l'erreur ponctuelle. Dans la plupart des cas, la solution de cette équation est bien connue (voir Tables 1 et 2). Seul le cas $r>0, s>0$ n'a pas été complètement résolu. C'est ce cas qui est étudié ici : les vitesses sont données explicitement dans le théorème 3.1 .

Trois cas sont à différencier : $r=s, r<s$ et $r>s$. Si $r$ est égal à $s$, les vitesses (ponctuelles et intégrées) sont en $n^{-a /(a+b)}$ modifiées par un facteur logarithmique. Si $r$ est différent de $s$, on observe un phénomène original : les vitesses dépendent de l'intervalle $] k /(k+1),(k+1) /(k+2)], k \in \mathbb{N}$ auquel appartient $r / s$ ou $s / r$. Si $r$ est strictement inférieur à $s$ (biais dominant), le terme principal est d'ordre $\exp \left[b_{0}(\ln n)^{r / s}\right]$ avec $b_{0}=-2 a /(2 b)^{r / s}$, et si $r$ est strictement supérieur à $s$ (variance dominante), le terme principal est d'ordre $\exp \left[-d_{0}(\ln n)^{s / r}\right] / n$ avec $d_{0}=-2 b /(2 a)^{s / r}$.

Ainsi ces vitesses originales décroissent plus vite que n’importe quelle fonction logarithmique. De plus elles sont optimales lorque les bornes inférieures correspondantes sont connues, c'est-à-dire $r=s=1$ 
(voir [11]) et $r<s$ (voir[2]). Il est à noter qu'étant donné la complexité de ces vitesses, il est réellement intéressant de construire des estimateurs adaptatifs, c'est-à-dire des estimateurs dont le risque atteint automatiquement les vitesses minimax.

\section{Introduction}

We consider the following deconvolution problem:

$$
Y_{i}=X_{i}+\varepsilon_{i} \quad i=1, \ldots, n
$$

where the $X_{i}$ 's are independent and identically distributed random variables with an unknown density $g$ and the random variables $\varepsilon_{i}$ are i.i.d with known density $f_{\varepsilon}$. Moreover $\left(X_{i}\right)$ and $\left(\varepsilon_{i}\right)$ are independent. The aim is to estimate $g$ from data $Y_{1}, \ldots, Y_{n}$.

The hypothesis framework is the following. Denote, for all function $u, u^{*}$ the Fourier transform of $u$ : $u^{*}(x)=\int e^{i x t} u(t) d t$. We suppose that noise is such that for all $x$ in $\mathbb{R}, f_{\varepsilon}^{*}(x) \neq 0$ and that it satisfies the following assumption:

N1. There exist $s \geq 0, b \geq 0, \gamma \in \mathbb{R}(\gamma>0$ if $s=0)$ and $k_{0}, k_{1}>0$ such that

$$
k_{0}\left(x^{2}+1\right)^{-\gamma / 2} \exp \left(-b|x|^{s}\right) \leq\left|f_{\varepsilon}^{*}(x)\right| \leq k_{1}\left(x^{2}+1\right)^{-\gamma / 2} \exp \left(-b|x|^{s}\right)
$$

We assume that $g$ belongs to the space

$$
\mathcal{A}_{\delta, r, a}(L)=\left\{g \text { is a probability density on } \mathbb{R} \text { and } \int\left|g^{*}(x)\right|^{2}\left(x^{2}+1\right)^{\delta} \exp \left(2 a|x|^{r}\right) \leq L\right\}
$$

with $r \geq 0, a \geq 0, \delta \in \mathbb{R}(\delta>1 / 2$ if $r=0), L>0$. When $r>0$ the function is known as supersmooth, and as ordinary smooth else. The terminology is the same for noise.

This problem has been extensively studied for a function $g$ belonging to a Sobolev or Hölder class (i.e. $r=0$ ): see among others [3], [5], [6,7], [8], [10]. The bad rates of convergence (power of $\ln n$ ) for supersmooth noise (and then in particular for Gaussian distributions) lead to consider supersmooth functions. First [9] and more recently [1], [2] and [4] studied estimators in this context.

The contribution of this paper is to provide exact and explicit rates of convergence, even in the case $r>0$ and $s>0$ where up to now the rates were not explicitly available except in very particular cases.

\section{Estimators and preliminar results}

\subsection{Estimators}

The classical kernel estimator is the following:

$$
\hat{g}_{n}(x)=\frac{1}{n h} \sum_{i=1}^{n} K\left(\frac{x-Y_{i}}{h}\right)
$$

where $K$ is the function defined as the inverse Fourier transform of $K^{*}(x)=\mathbb{1}_{\{|x| \leq 1\}} / f_{\varepsilon}^{*}(x / h)$. The pointwise mean squared error (denoted by MSE) and mean integrated squared error (denoted by MISE) are established in [2]: 
Proposition 2.1 If $g$ belongs to $\mathcal{A}_{\delta, r, a}(L)$, then under Assumption N1,

$$
\begin{aligned}
& M I S E=\mathbb{E}\left\|g-\hat{g}_{n}\right\|_{2}^{2}=O\left(h^{2 \delta} \exp \left(-2 a / h^{r}\right)+\frac{h^{s-1-2 \gamma}}{n} \exp \left(2 b / h^{s}\right)\right) \quad \text { and } \\
& M S E=\mathbb{E}\left|g(x)-\hat{g}_{n}(x)\right|^{2}=O\left(h^{2 \delta+r-1} \exp \left(-2 a / h^{r}\right)+\min \left(1, h^{s-1}\right) \frac{h^{s-1-2 \gamma}}{n} \exp \left(2 b / h^{s}\right)\right)
\end{aligned}
$$

This estimator has the advantage to be optimal in the sharp asymptotic minimax sense (see [2]) but provides an adaptive estimator only in particular cases. That is why we present the projection estimator introduced in [4].

Let $\varphi(x)=\sin (\pi x) /(\pi x)$ and $\varphi_{m, j}(x)=\sqrt{L_{m}} \varphi\left(L_{m} x-j\right)$. Consider $u_{t}(x)=(1 / 2 \pi)\left(t^{*}(-x) / f_{\varepsilon}^{*}(x)\right)$. Then the projection estimator is defined by

$$
\hat{g}_{m}(x)=\sum_{|j| \leq K_{n}} \hat{a}_{m, j} \varphi_{m, j} \quad \text { where } \hat{a}_{m, j}=\frac{1}{n} \sum_{i=1}^{n} u_{\varphi_{m, j}}^{*}\left(Y_{i}\right)
$$

For this estimator, the following result is proved in [4]:

Proposition 2.2 Assume that $f_{\varepsilon} \in L^{2}$ (i.e. $\gamma>1 / 2$ when $s=0$ ) and that $g$ is a $L^{2}$ function which verifies $\int x^{2} g^{2}(x) d x \leq M$. If $g$ belongs to $\mathcal{A}_{\delta, r, a}(L)$, then under Assumption N1,

$M I S E=\mathbb{E}\left\|g-\hat{g}_{m}\right\|_{2}^{2}=O\left(L_{m}^{-2 \delta} \exp \left(-2 a \pi^{r} L_{m}^{r}\right)+\frac{L_{m}^{2 \gamma+1-s}}{n} \exp \left(2 b \pi^{s} L_{m}^{s}\right)\right)$

Then, an adaptive estimator can be defined using a model selection method (see [4] for details).

\subsection{Rates of convergence}

We can observe that both estimators have the same $L^{2}$ rate of convergence (take $h^{-1}=\pi L_{m}$ ). To compute this rate, we have to minimize the risk orders in $h$ (or $L_{m}$ ). By setting to zero the derivative of this quantity we obtain the equation

$$
\exp \left(\frac{2 b}{h^{s}}+\frac{2 a}{h^{r}}\right) h^{\alpha}=O(n)
$$

where $\alpha=r-2 \delta-2 \gamma-1$ if we consider the integrated error and $\alpha=-2 \delta-2 \gamma+(s-1)_{+}$if we consider the pointwise error. In most cases, the solution of this equation is well known and leads to the following tables where different regularities for $g$ and $f_{\varepsilon}$ are examined:

\begin{tabular}{ccc}
\cline { 2 - 3 } & $s=0$ & $s>0$ \\
\hline$r=0$ & $n^{-\frac{2 \delta}{2 \delta+2 \gamma+1}}$ & $(\ln n)^{-\frac{2 \delta}{s}}$ \\
\hline$r>0$ & $\frac{(\ln n)^{\frac{2 \gamma+1}{r}}}{n}$ & Theorem 3.1 \\
\hline
\end{tabular}

Table 1

Rates of convergence for the MISE.

\begin{tabular}{ccc}
\cline { 2 - 3 } & $s=0$ & $s>0$ \\
\hline$r=0$ & $n^{\frac{1-2 \delta}{2 \delta+2 \gamma}}$ & $(\ln n)^{\frac{1-2 \delta}{s}}$ \\
\hline$r>0$ & $\frac{(\ln n)^{\frac{2 \gamma+1}{r}}}{n}$ & Theorem 3.1 \\
\hline
\end{tabular}

Table 2

Rates of convergence for the MSE.

Except for the bottom right cells (to be completed in the next section), these rates are known to be optimal minimax rates: see [6] and [1] for the lower bounds. 


\section{Results}

The rates of convergence in the case $(r>0, s>0)$ depend on the integer $k$ such that $r / s$ or $s / r$ belongs to the interval $(k /(k+1),(k+1) /(k+2)]$ :

Theorem 3.1 We assume $r>0$ and $s>0$. Let $k \in \mathbb{N}$ and $\lambda=\mu^{-1}=r / s$. Then

- if $r=s$, if $\xi=[2 \delta b+(s-2 \gamma-1) a] /[(a+b) s]$

$$
\begin{gathered}
M I S E=O\left(n^{-a /(a+b)}(\ln n)^{-\xi}\right) ; \\
M S E=O\left(n^{-a /(a+b)}(\ln n)^{-\xi+\frac{(1-s)+b}{(a+b) s}}\right)
\end{gathered}
$$

- if $r<s$ and $\frac{k}{k+1}<\lambda \leq \frac{k+1}{k+2}$, there exist reals $b_{i}$ such that

$$
\begin{gathered}
M I S E=O\left((\ln n)^{-2 \delta / s} \exp \left[\sum_{i=0}^{k} b_{i}(\ln n)^{(i+1) \lambda-i}\right]\right) \\
M S E=O\left((\ln n)^{(-2 \delta-r+1) / s} \exp \left[\sum_{i=0}^{k} b_{i}(\ln n)^{(i+1) \lambda-i}\right]\right)
\end{gathered}
$$

- if $r>s$ and $\frac{k}{k+1}<\mu \leq \frac{k+1}{k+2}$, there exist reals $d_{i}$ such that,

$$
\begin{gathered}
M I S E=O\left(\frac{(\ln n)^{(1+2 \gamma-s) / r}}{n} \exp \left[-\sum_{i=0}^{k} d_{i}(\ln n)^{(i+1) \mu-i}\right]\right) ; \\
M S E=O\left(\frac{(\ln n)^{\left(1+2 \gamma-s-(s-1)_{+}\right) / r}}{n} \exp \left[-\sum_{i=0}^{k} d_{i}(\ln n)^{(i+1) \mu-i}\right]\right)
\end{gathered}
$$

The coefficients $b_{i}$ and $d_{i}$ are computable, see Section 4 for the exact form of reals $b_{i}$. Notice that these original rates have the property to decrease faster than any logarithmic function. Moreover, they are optimal in the cases where the corresponding lower bounds are known, i.e $r=s=1$ (see [11]) and $r<s$ (see[2]). We can also remark that, given the complexity of these rates, it is woth finding adaptive estimators, i.e. estimators whose risk automatically achieves the minimax rates.

\section{Proof of Theorem 3.1}

- If $r=s$, we check that $h^{*}=(2 a+2 b)^{1 / s}\left(\ln n+\frac{\alpha}{s} \ln \ln n\right)^{-1 / s}$ satisfies Equation (1). The corresponding risks are easily obtained.

- If $r<s$ and $\frac{k}{k+1}<\lambda \leq \frac{k+1}{k+2}$, let $h *=(2 b)^{1 / s}\left[\ln n+\frac{\alpha}{s} \ln \ln n+\sum_{i=0}^{k} b_{i}(\ln n)^{(i+1) \lambda-i}\right]^{-1 / s}$

$$
\begin{aligned}
\exp \left[\frac{2 B}{h^{* s}}+\frac{2 A}{h^{* r}}+\alpha \ln h^{*}\right]= & K n \exp \left[\frac{2 a}{(2 b)^{\lambda}}(\ln n)^{\lambda}\left(1+\lambda u_{n}+. .+\frac{\lambda(\lambda-1) . .(\lambda-k)}{(k+1) !} u_{n}^{k+1}+o\left(u_{n}^{k+1}\right)\right)\right. \\
& \left.+\sum_{i=0}^{k} b_{i}(\ln n)^{(i+1) \lambda-i}-\frac{\alpha}{s} \ln (1+o(1))\right]
\end{aligned}
$$


with $u_{n}:=\frac{\alpha}{s} \frac{\ln \ln n}{\ln n}+\sum_{i=0}^{k} b_{i}(\ln n)^{(i+1) \lambda-(i+1)}$

By noting that for $2 \leq j \leq k+1, u_{n}^{j}=\sum_{i=j}^{k+1} \sum_{p_{1}+. .+p_{j}=i} b_{p_{1}-1} . . b_{p_{j}-1}(\ln n)^{(\lambda-1) i}+o\left((\ln n)^{(\lambda-1)(k+1)}\right)$, we compute

$$
\exp \left[\frac{2 b}{h^{* s}}+\frac{2 a}{h^{* r}}+\alpha \ln h^{*}\right]=K n \exp \left[o(1)+\sum_{i=0}^{k+1} M_{i}(\ln n)^{(i+1) \lambda-i}+o\left((\ln n)^{(k+2) \lambda-(k+1)}\right)\right]
$$

with $M_{0}=b_{0}+\frac{2 a}{(2 b)^{\lambda}}$ and for $1 \leq i \leq k+1, M_{i}=b_{i}+\frac{2 a}{(2 b)^{\lambda}} \sum_{j=1}^{i} \frac{\lambda \ldots(\lambda-j+1)}{j !} \sum_{p_{1}+. .+p_{j}=i} b_{p_{1}-1} . . b_{p_{j}-1}$ by denoting $b_{k+1}=0$. The $b_{i}$ 's are recursively defined by $M_{0}=\ldots=M_{k}=0$ and thus Equation (1) is verified. The risks are then computed by using the same tools.

- The reasoning is the same in the case $r>s$

\section{References}

[1] C. Butucea, Deconvolution of supersmooth densites with smooth noise, Canad. J. Statist. 32 (2004) 181-192.

[2] C. Butucea and A.B. Tsybakov, Sharp optimality for density deconvolution with dominating bias, Theory Probab. Appl. (2006) to appear.

[3] R.J. Carrol and P. Hall, Optimal rates of convergence for deconvolving a density, J. Amer. Statist. Assoc. 83 (1988) $1184-1186$

[4] F. Comte, Y. Rozenholc and M.-L. Taupin, Penalized contrast estimator for adaptive density deconvolution, Canad. J. Statist. 34 (2006) to appear.

[5] L. Devroye, Consistent deconvolution in density estimation, Canad. Journ. Statist. 17 (1989) 235-239.

[6] J. Fan, On the optimal rates of convergence for nonparametric deconvolution problem, Ann. Statist. 19 (1991) 1257-1272.

[7] J. Fan, Adaptatively local one-dimensional subproblems with application to a deconvolution problem, Ann. Statist. 21 (1993) 600-610.

[8] M.C. Liu and R.L. Taylor, A consistent non-parametric density estimator for the deconvolution problem, Canad. Journ. Statist. 17 (1989) 427-438.

[9] M. Pensky and B. Vidakovic, Adaptative wavelet estimator for nonparametric density deconvolution, Ann. Statist. 27 (1999) 2033-2053.

[10] L. Stefanski, Rates of convergence of some estimators in a class of deconvolution problems, Statist. Prob. Lett. 9 (1990) 229-235.

[11] A.B. Tsybakov, On the best rate of adaptive estimation in some inverse problems, C. R. Acad. Sci. Paris Sér. I 330 (2000) 835-840. 